\title{
New Exact Solutions for the Variable Coefficient Two-Dimensional Burger Equation
}

\author{
Rehab M. EI -Shiekh
}

Department of Mathematics, Faculty of Education, Ain Shams University, Heliopolis, Cairo, Egypt

\begin{abstract}
In this paper, the variable coefficient two-dimensional Burger equation is studied by two distinct methods. The Exp-function method with the aid of symbolic computation is used to derive soliton solutions of this equation. The $\frac{G^{\prime}}{G}$ - expansion method is used also to construct travelling wave solutions for the variable coefficient two-dimensional Burger equation with the aid of symbolic computation. The travelling wave solutions are expressed by the hyperbolic, the trigonometric functions and rational functions. The study highlights the significant features of the employed methods and its capability of handling exact solutions for the variable coefficient two-dimensional Burger equation without any restrictions on the form of the variable coefficient. The obtained solutions are considered new with the comparison of other solutions obtained before.
\end{abstract}

Keywords The Exp-function Method; The $\frac{G^{\prime}}{G}$ - -Expansion Method; The Variable Coefficient Two-Dimensional Burger Equation; New Exact Solutions

\section{Introduction}

An evolution equation usually means a partial differential equation with one of the independent variables being time $t$. There are many nonlinear evolution equations arising from physics, mechanics, biology, chemistry, material science and plasma physics etc. indeed in this paper we confine our attention to the variable coefficients nonlinear evolution equations since they are able to model the real world in many fields of physical and engineering science although their coefficient functions often make the studies very hard also they covers the constant coefficients case by assuming that the coefficient functions constants. One of the variable coefficients nonlinear evolution equations is the variable coefficient two-dimensional Burger equation

$$
\left(u_{t}+u u_{x}-u_{x x}\right)_{x}+s(t) u_{y y}=0
$$

Equation (1.1) with $\mathrm{s}=$ constant is sometimes referred to as Zabolotskaya-Khokhlov equation in nonlinear acoustics[1,2]. Painlevé analysis of the constant coefficient version of (1.1) was carried out in[3]. The authors showed that the equation possesses the conditional painlevé property and obtained its exact solutions by use of truncation. Also Moussa et al in[4] have applied the symmetry method on Zabolotskaya-Khokhlov equation and obtained new exact solutions for it. Güngör in[5-6] used the symmetry method

* Corresponding author:

rehab_el_shiekh@yahoo.com (Rehab M. El -Shiekh)

Published online at http://journal.sapub.org/ajcam

Copyright (C) 2012 Scientific \& Academic Publishing. All Rights Reserved to find similarity reductions for Eq. (1.1) with it's variable coefficient $s(t)$ but those solutions obtained for only some forms of $s(t)$.

\section{The Exp-function Method}

To illustrate the basic idea of the Exp-function method[7], we consider the following nonlinear evolution equations with only three independent variables

$$
P\left(u, u_{t}, u_{x}, u_{y}, u_{x x}, u_{y y}, u_{x y}\right)=0,
$$

where $\mathrm{P}$ is a polynomial function with respect to the indicated variables or some function can be reduce to a polynomial function by using some transformation.

Making use of the travelling wave transformation

$$
u=u(\xi), \quad \xi=t+c_{1} x+c_{2} y,
$$

Where $c_{1}$ and $c_{2}$ are arbitrary constants to be determined later Then Eq. (2.1) reduces to an ordinary differential equation

$$
\Psi\left(u, u^{\prime}, c_{1} u^{\prime}, c_{2} u^{\prime}, c_{1}^{2} u^{\prime \prime}, c_{2}^{2} u^{\prime \prime}, \ldots\right)=0
$$

The Exp-function method is based on the assumption that the travelling wave solution of the previous equation can be expressed in the following form

$$
u(\xi)=\frac{\sum_{n=-c}^{d} a_{n} \exp (n \xi)}{\sum_{m=-p}^{q} b_{m} \exp (m \xi)}
$$

where $\mathrm{c} ; \mathrm{d} ; \mathrm{p}$ and $\mathrm{q}$ are unknown positive integers, $a_{n}$ and $b_{n}$ are unknown constants. To determine $\mathrm{c}$ and $\mathrm{p}$ we 
balance the linear term of the highest order in (2.3) with the highest order nonlinear term.

Similarly, we can determine $\mathrm{d}$ and $\mathrm{q}$ by balancing the linear term of the lowest order in (2.3) with the lowest order nonlinear term.

\section{The $\frac{G^{\prime}}{G}$ - expansion Method}

The same assumptions as given before in Eqs. (2.1-2.3) are used but we assume that the solution of Eq. (2.3) takes the following form[8]

$$
u=\sum_{i=0}^{m} A_{i}\left(\frac{G^{\prime}}{G}\right)^{i},
$$

where $G=G(\xi)$ satisfy a second order linear differential equation

$$
G^{\prime \prime}+\lambda G^{\prime}+\mu G=0
$$

where $_{G^{\prime}}=\frac{d G(\xi)}{d \xi}, G^{\prime \prime}=\frac{d^{2} G(\xi)}{d \xi^{2}}, \quad \lambda$ and $\mu$ are constants to be determine later. The integer $\mathrm{m}$ can be determine by considering the homogeneous balance between the highest derivatives and the highest order nonlinear terms appearing in Eq. (2.3). Then by substituting Eq. (3.1) along with Eq. (3.2) into Eq. (2.3), collecting all terms with the same order of $\left(\frac{G^{\prime}}{G}\right)$ together, the left hand side of Eq. (2.3) is converted into another polynomial in $\left(\frac{G^{\prime}}{G}\right)$. Equating each coefficient of this polynomial to zero, yield a set of algebraic equations for $A_{i}(i=1,2, \ldots, m), \lambda, \mu, c_{1}$ and $c_{2}$ which can be solved by using Maple program, along with the general solutions of Eq. (3.1).

\section{Solutions of Equation (1.1) by using Exp-function Method}

Let $u=u(\xi)$, where $\xi=c_{1} x+c_{2} y+\int \tau(t) d t$

Eq. (1.1) becomes

$$
c_{1}\left(\tau(t) u^{\prime}+c_{1} u u^{\prime}-c_{1}^{2} u^{\prime \prime}\right)^{\prime}+s(t) c_{2}^{2} u^{\prime}=0,
$$

Integrate Eq. (4.1) with respect to $\xi$ twice, we get

$$
\left(c_{1} \tau(t)+c_{2}^{2} s(t)\right) u+\frac{c_{1}^{2}}{2} u^{2}-c_{1}^{3} u^{\prime}=0 .
$$

By re-writing Eq. (2.4) in an alternative form as follows:

$$
u(\xi)=\frac{a_{c} \exp (c \xi)+\ldots+a_{-d} \exp (-d \xi)}{b_{p} \exp (p \xi)+\ldots+b_{-q} \exp (-q \xi)} .
$$

In order to determine values of $\mathrm{c}$ and $\mathrm{p}$, we balance the linear term $\mathrm{u}^{\prime}$ of the highest order in Eq. (4.3) with the highest order nonlinear term $\mathrm{u}^{2}$ and we have

$$
\begin{aligned}
u^{\prime} & =\frac{C_{1} \exp [(c+p) \xi]+\ldots}{C_{2} \exp [2 p \xi]+\ldots} \\
u^{2} & =\frac{C_{3} \exp [2 c \xi]+\ldots}{C_{4} \exp [2 p \xi]+\ldots},
\end{aligned}
$$

Where $C_{i}$ are constant coefficients for simplicity. By balancing the highest order of Exp-function in Eqs. (4.4) and (4.5), we have $\mathrm{c}+\mathrm{p}=2 \mathrm{c}$, which leads to the limit

$$
\mathrm{p}=\mathrm{c}
$$

Proceeding the same manner as illustrated above, we can determine values of $d$ and $q$. Balancing the linear term of lowest order in Eq. (4.3)

$$
\begin{aligned}
& u^{\prime}=\frac{\ldots+d_{1} \exp [-(d+q) \xi]}{\ldots .+d_{2} \exp [-2 q \xi]} \\
& u^{2}=\frac{\ldots .+d_{3} \exp [-2 d \xi]}{\ldots .+d_{4} \exp [-2 q \xi]}
\end{aligned}
$$

where $d_{i}$ are determined coefficients only for simplicity, we have $-(d+q)=-2 d$, which leads to results

$$
d=q
$$

Case 1. $\mathrm{p}=\mathrm{c}=1$ and $\mathrm{d}=\mathrm{q}=1$, then solution of Eq. (4.3) takes the following form.

$$
u=\frac{a_{1} \exp (\xi)+a_{0}+a_{-1} \exp (-\xi)}{b_{1} \exp (\xi)+b_{0}+b_{-1} \exp (-\xi)}
$$

By substituting from Eq. (4.10) into Eq. (4.3) and equating the coefficients of the Exp-functions to zero we obtain the following algebraic system

$$
\begin{aligned}
& 2 c_{2}^{2} a_{-1} b_{-1} s(t)+c_{1}^{2} a_{-1}^{2}+2 c_{1} a_{-1} b_{-1} \tau(t)=0, \\
& c_{1}^{2} a_{1}^{2}+2 c_{1} a_{1} b_{1} \tau(t)+2 c_{2}^{2} a_{1} b_{1} s(t)=0, \\
& 2 c_{1}^{2} a_{0} a_{-1}-2 c_{1}^{3} a_{0} b_{-1}+2 c_{2}^{2} a_{-1} b_{0} s(t)+2 c_{1} a_{-1} b_{0} \tau(t) \\
& +2 c_{2}^{2} a_{0} b_{-1} s(t)+2 c_{1} a_{0} b_{-1} \tau(t)+2 c_{1}^{3} a_{-1} b_{0}=0, \\
& 2 c_{1}^{3} b_{0} a_{1}-2 c_{1} a_{0} b_{1} \tau(t)-2 c_{1} a_{1} b_{0} \tau(t)-2 a_{0} b_{1} c_{2}^{2} s(t) \\
& -2 c_{2}^{2} a_{1} b_{0} s(t)-2 c_{1}^{3} a_{0} b_{1}-2 c_{1}^{2} a_{0} a_{1}=0, \\
& 4 c_{1}^{3} a_{1} b_{-1}-2 c_{2}^{2} a_{0} b_{0} s(t)-2 c_{2}^{2} a_{1} b_{-1} s(t)-2 c_{1}^{2} a_{1} a_{-1} \\
& -c_{1}^{2} a_{0}^{2}-4 c_{1}^{3} a_{-1} b_{-1}-2 c_{1} a_{0} b_{0} \tau(t) \\
& 2 c_{1} a_{1} b_{-1} \tau(t)+2 c_{1} a_{-1} b_{1} \tau(t)+2 c_{2}^{2} a_{-1} b_{1} s(t)=0 .
\end{aligned}
$$

Solving the system of algebraic equations (4.11) with the aid of Maple, we obtain

$$
a_{-1}=\frac{a_{1} b_{-1}}{b_{1}}, a_{0}=\frac{a_{1} b_{0}}{b_{1}}, \tau(t)=-\frac{a_{1} c_{1}}{2 b_{1}}-\frac{c_{2}^{2}}{c_{1}} s(t)
$$

by back substitution we get the following new exact solution for the variable coefficient two-dimensional Burger equation

$$
\begin{aligned}
& u_{1}=\frac{a_{1} \exp (\xi)+\frac{a_{1} b_{0}}{b_{1}}+\frac{a_{1} b_{-1}}{b_{1}} \exp (-\xi)}{b_{1} \exp (\xi)+b_{0}+b_{-1} \exp (-\xi)}, \\
& \text { where } \xi=\mathrm{c}_{1} x+c_{2} y-\int\left(\frac{a_{1} c_{1}}{2 b_{1}}+\frac{c_{2}^{2}}{c_{1}} s(t)\right) d t, \\
& \text { and } a_{1}, b_{0}, b_{1} \text {, and } b_{2} \text { are arbitrary constants }
\end{aligned}
$$

Case 2. $\mathrm{p}=\mathrm{c}=2$ and $\mathrm{d}=\mathrm{q}=1$, then Eq. (4.3) has the following solution for

$$
u=\frac{a_{2} \exp (2 \xi)+a_{1} \exp (\xi)+a_{0}+a_{-1} \exp (-\xi)}{b_{2} \exp (2 \xi)+b_{1} \exp (\xi)+b_{0}+b_{-1} \exp (-\xi)}
$$

Substituting Eq. (4.13) into Eq. (4.3). Equating to zero the coefficients of all powers of $\exp (n \xi)$ yields a set of algebraic equations for $a_{2}, a_{1}, a_{0}, a_{-1}, b_{2}, b_{1}, b_{0}, b_{-1}, c_{1}$ and $c_{2}$. Solving this system of algebraic equations with the aid of Maple, we 
obtain two sets of solutions

$$
\begin{aligned}
& \text { set } 1: a_{-1}=0, a_{1}=\frac{2 c_{1}\left(a_{2} b_{-1}-a_{0} b_{1}\right)}{a_{0}}, b_{2}=-\frac{a_{2}}{2 c_{1}}, b_{0} \\
& =\frac{-a_{0}^{3}+4 c_{1}^{2} b_{-1}\left(a_{2} b_{-1}-a_{0} b_{1}\right)}{2 a_{0}{ }^{2} c_{1}}, \tau(t)=c_{1}^{2}-\frac{c_{2}^{2}}{c_{1}} s(t) . \\
& \text { set2: } a_{2}=0, a_{0}=\frac{2 c_{1}\left(a_{1} b_{0}-a_{-1} b_{2}\right)}{a_{1}}, b_{1}=\frac{4 c_{1}^{2} b_{2}\left(a_{1} b_{0}-b_{2} a_{-1}\right)+a_{1}^{3}}{2 a_{1}{ }^{3} c_{1}} \\
& , b_{-1}=\frac{a_{-1}}{2 c_{1}}, \tau(t)=-c_{1}{ }^{2}-\frac{c_{2}{ }^{2}}{c_{1}} s(t)
\end{aligned}
$$

Therefore the variable coefficient two-dimensional Burger equation has the following solitary wave solutions

$$
u_{2}=\frac{a_{2} \exp (2 \xi)+\frac{2 c_{1}\left(a_{2} b_{-1}-a_{0} b_{1}\right)}{a_{0}} \exp (\xi)+a_{0}}{-\frac{a_{2}}{2 c_{1}} \exp (2 \xi)+b_{1} \exp (\xi)+\frac{-a_{0}^{3}+4 c_{1}^{2} b_{-1}\left(a_{2} b_{-1}-a_{0} b_{1}\right)}{2 a_{0}^{2} c_{1}}+b_{-1} \exp (-\xi)}
$$

where $\xi=c_{1} x+c_{2} y+\int\left(c_{1}^{2}-\frac{c_{2}^{2}}{c_{1}} s(t)\right) d t, \quad$ and $\mathrm{a}_{0}, a_{2}, b_{1}$ and $b_{-1}$ are constants.

$$
\begin{gathered}
u_{3}=\frac{a_{1} \exp (\xi)+2 c_{1}\left(a_{1} b_{0}-a_{-1} b_{2}\right)\left(a_{1}\right)^{-1}}{b_{2} \exp (2 \xi)+\left(4 c_{1}^{2} b_{2}\left(a_{1} b_{0}-b_{2} a_{-1}\right)+a_{1}^{3}\right)\left(2 a_{1}^{2} c_{1}\right)^{-1} \exp (\xi)} \\
+b_{0}+a_{-1}\left(2 c_{1}\right)^{-1} \exp (-\xi)
\end{gathered}
$$

where $\xi=c_{1} x+c_{2} y-\int\left(c_{1}^{2}+\frac{c_{2}^{2}}{c_{1}} s(t)\right) d t$, and $\mathrm{a}_{1}, a_{-1}, b_{0}$ and $b_{2}$ are constants.

Case 3. $p=c=2$ and $d=q=2$, by the same manner as we have done in the previous two cases we obtain the following exact solution for the variable coefficient two-dimensional Burger equation

$$
\begin{aligned}
& u_{4}=\left[a_{2} c_{1} a_{-1}^{2} \exp (2 \xi)+2 c_{1}^{2}\left(a_{2} b_{-2}-a_{-1} b_{1}\right) \exp (\xi)\right. \\
& \left.+a_{0} c_{1} a_{-1}^{2}+c_{1} a_{-1}^{3} \exp (-\xi)\right] /\left[-\frac{a_{2}}{2} c_{1} a_{-1}^{2} \exp (2 \xi)\right. \\
& +c_{1} a_{-1}^{2} b_{1} \exp (\xi)+4 c_{1}^{2} b_{-2}\left(b_{-2} a_{2}-a_{-1} b_{1}\right)-a_{0} a_{-1}^{2}- \\
& \left.\left(a_{-1}{ }^{3}-2 a_{-1} c_{1} a_{0} b_{-2}\right) \exp (-\xi)+2 c_{1} a_{-1}^{2} b_{-2} \exp (-2 \xi)\right]
\end{aligned}
$$

Where $\xi=c_{1} x+c_{2} y+\int\left(c_{1}^{2}-\frac{c_{2}^{2}}{c_{1}} s(t)\right) d t$ and $\mathrm{a}_{0}, a_{-1}, a_{2}, b_{1}$ and $b_{-2}$ are constants.

\section{New Solutions for Equation (1.1) by using $G$-expansion Method}

By substitution from Eq. (3.1) in (4.3) and balancing the nonlinear term $u^{2}$ with the linear $u^{\prime}$ yield that the leading order $m=1$, therefore

$$
u(\xi)=A_{0}+A_{1}\left(\frac{G^{\prime}}{G}\right) .
$$

By substitution from (5.1) in (4.2) and by using (3.2), then
Eq. (4.2) becomes a polynomial in $\left(\frac{G^{\prime}}{G}\right)$. Equating the coefficients of $\left(\frac{G^{\prime}}{G}\right)$ by zero yields the following algebric system

$$
\begin{array}{ll}
\left(\frac{G^{\prime}}{G}\right)^{2}: & A_{1}+2 c_{1}=0, \\
\left(\frac{G^{\prime}}{G}\right): & c_{1} \tau(t)+c_{2}{ }^{2} s(t)+c_{1}{ }^{2} A_{0}+\lambda c_{1}^{3}=0, \\
\left(\frac{G^{\prime}}{G}\right)^{0}: & A_{0}\left(c_{1} \tau(t)+c_{2}{ }^{2} s(t)\right)+\frac{c_{1}^{2}}{2} A_{0}{ }^{2}+c_{1}^{3} \mu A_{1}=0 .
\end{array}
$$

Solving the previous system yields the following solutions for it

$$
A_{0}=c_{1}\left(-\lambda \pm \sqrt{\lambda^{2}-4 \mu}\right), \quad \tau(t)=\mp c_{1}^{2} \sqrt{\lambda^{2}-4 \mu}-\frac{c_{2}^{2}}{c_{1}} s(t)
$$

From (5.3) into (5.1) and by using (4.1), we get the following rational and periodic solutions for the variable coefficient two-dimensional Burger equation

Case 1: $\lambda^{2}-4 \mu>0$

$$
\begin{aligned}
& u_{5}=c_{1} \sqrt{\lambda^{2}-4 \mu}\left(\frac{k_{1} \sinh (\xi)+k_{2} \cosh (\xi)}{k_{1} \cosh (\xi)+k_{2} \sinh (\xi)} \pm 1\right) \\
& \text { where } \xi=\frac{\sqrt{\lambda^{2}-4 \mu}}{2}\left(c_{1} x+c_{2} y+\int\left(\mp c_{1}^{2} \sqrt{\lambda^{2}-4 \mu}-\frac{c_{2}^{2}}{c_{1}} s(t)\right) d t\right)
\end{aligned}
$$

If $k_{1}=0$, then we get

$u_{6}=c_{1} \sqrt{\lambda^{2}-4 \mu}$ coth

$$
\left(\frac{\sqrt{\lambda^{2}-4 \mu}}{2}\left(c_{1} x+c_{2} y+\int\left(\mp c_{1}^{2} \sqrt{\lambda^{2}-4 \mu}-\frac{c_{2}^{2}}{c_{1}} s(t)\right) d t\right) \pm 1\right) \text {, }
$$

Also, if we put $k_{2}=0$ we obtain the following new solution

$$
\begin{aligned}
& u_{7}=c_{1} \sqrt{\lambda^{2}-4 \mu} \text { tanh } \\
& \left(\frac{\sqrt{\lambda^{2}-4 \mu}}{2}\left(c_{1} x+c_{2} y+\int\left(\mp c_{1}^{2} \sqrt{\lambda^{2}-4 \mu}-\frac{c_{2}^{2}}{c_{1}} s(t)\right) d t\right) \pm 1\right),
\end{aligned}
$$

Case 2: $\lambda^{2}-4 \mu<0$

$$
\begin{aligned}
& u_{8}=c_{1} \sqrt{4 \mu-\lambda^{2}}\left(\frac{-k_{1} \sin (\xi)+k_{2} \cos (\xi)}{k_{1} \cos (\xi)+k_{2} \sin (\xi)} \pm 1\right), \\
& \text { where } \xi=\frac{\sqrt{4 \mu-\lambda^{2}}}{2}\left(c_{1} x+c_{2} y+\int\left(\mp c_{1}^{2} \sqrt{\lambda^{2}-4 \mu}-\frac{c_{2}^{2}}{c_{1}} s(t)\right) d t\right)
\end{aligned}
$$

If $k_{1}=0$, then we get

$$
\begin{aligned}
& u_{9}=c_{1} \sqrt{4 \mu-\lambda^{2}} \cot \\
& \left(\frac{\sqrt{4 \mu-\lambda^{2}}}{2}\left(c_{1} x+c_{2} y+\int\left(\mp c_{1}^{2} \sqrt{\lambda^{2}-4 \mu}-\frac{c_{2}^{2}}{c_{1}} s(t)\right) d t\right) \pm 1\right)
\end{aligned}
$$

Also, if we put $k_{2}=0$ we obtain the following new exact solution

$$
\begin{aligned}
& u_{10}=-c_{1} \sqrt{4 \mu-\lambda^{2}} \text { tan } \\
& \left(\frac{\sqrt{4 \mu-\lambda^{2}}}{2}\left(c_{1} x+c_{2} y+\int\left(\mp c_{1}^{2} \sqrt{\lambda^{2}-4 \mu}-\frac{c_{2}{ }^{2}}{c_{1}} s(t)\right) d t\right) \pm 1\right),
\end{aligned}
$$

In equations from (5.4)-(5.8) $k_{1}$ and $k_{2}$ are arbitrary constants. 


\section{Conclusions}

In this paper, we have applied the Exp-function method and the $\frac{G^{\prime}}{G}$-expansion method on the variable coefficient two-dimensional Burger equation and many new exact solutions in the form of exp-function, hyperbolic, trigonometric functions and rational functions without any restructions on the variable coefficient $\mathrm{s}(\mathrm{t})$ which makes those solutions new and not obtained before.

\section{REFERENCES}

[1] Zabolotskaya E. A., Khokhlov R. V., Sov. phys. Acoust. 15 (1969) 35

[2] Kuznetsov V. P., Sov. Pys. Acoust. , 16 (1971) 467

[3] Webb G. M. , Zank G. P. J. Phys. A: Math. Gen. 23 (1990) 5465

[4] Moussa M. H. M., El Shikh M. Rehab, Physica A 371 (2006) 325

[5] Güngör F., J. Phys. A: Math. Gen. 34 (2001) 4313

[6] Güngör F., J. Phys. A: Math. Gen. 35 (2002) 1805

[7] Sheng Zhang, Phys. Lett. A 371 (2007) 65

[8] Ahmet Bekir, Phys. Lett. A, 372 (2008) 3400 